\title{
Liver disease in infancy: histological features and relationship to $\alpha_{1}$-antitrypsin phenotype
}

\author{
I. C. TALBOT ${ }^{1}$ AND A. P. MOWAT \\ From the Departments of Morbid Anatomy and Child Health, King's College Hospital, London
}

SYNOPSIS Sixty-nine specimens of liver tissue from 53 infants with neonatal hepatitis or its sequelae were examined without knowledge of the $a_{1}$-antitrypsin phenotype. Distinctive, diastase-resistant, PAS-positive, pure magenta-coloured, sharply defined globules, 2-20 microns in diameter were found in periportal and paraseptal hepatocytes in all liver biopsies from eight $\alpha_{1}$-antitrypsin deficient (PiZZ) infants biopsied after the age of 12 weeks. Such globules were not seen in biopsies from PiZZ individuals aged less than 12 weeks nor in individuals of normal $a_{1}$-antitrypsin phenotype (PiMM). No other specific histological abnormality was found in PiZZ individuals, but in them giant-cell transformation was infrequent and liver damage was more severe, three of 14 cases developing cirrhosis in contrast to four of 27 PiMM subjects. The pathogenesis of liver disease in $\mathrm{PiZZ}$ individuals is discussed.

Genetic deficiency of the serum protein $\alpha_{1}$-antitrypsin, a protease inhibitor which accounts for $90 \%$ of serum $\alpha_{1}$-globulin, is an inborn error of metabolism inherited in an autosomal recessive fashion. By starch gel electrophoresis $\alpha_{1}$-antitrypsin can be shown to be polymorphic, the different alleles of the protease inhibitor $(\mathrm{Pi})$ appearing as distinct bands which have been named so that their relative alphabetical order indicates their electrophoretic mobility on acid starch gel. Normal individuals have $\mathrm{M}$ bands and the Pi phenotype $\mathrm{MM}$ whereas deficient individuals have $Z$ bands and the PiZZ phenotype (Brunt, 1974). Although $\alpha_{1}$-antitrypsin inhibits trypsin and other proteolytic enzymes in vitro, its in vivo function is unknown.

Subjects who are congenitally deficient in $\alpha_{1}$-antitrypsin (PiZZ) may have no associated disease, may develop emphysema in early life (Hutchison, Cook, Barter, Harris, and Hugh-Jones, 1971) or may develop significant liver disease in childhood. $\alpha_{1}$-Antitrypsin deficiency ( $\alpha_{1}$-ATD) was first associated with hepatic cirrhosis in children by Sharp, Bridges, Krivit, and Freier (1969). The pathogenetic link between deficiency of the anti-protease and cirrhosis is uncertain. The liver disease frequently presents as an acute hepatitis with conjugated hyperbilirubinaemia and features of hepatocellular

'Present address: Pathology Department, St Mark's Hospital, City Road, London ECIV 2PS

Received for publication 3 March 1975. damage such as raised serum transaminase and often bilirubinuria, pale stools, and hepatosplenomegaly (Cottrall, Cook, and Mowat, 1974). After the acute hepatitis settles liver function tests remain abnormal (Porter, Mowat, Cook, Haynes, Shilkin, and Williams, 1972) and by the second decade of life many of the children develop cirrhosis and its complications (Sharp, 1971; Brunt, 1974). A minority present with cirrhosis without antecedent neonatal liver disease. Thirty-four out of $40 \mathrm{PiZZ}$ infants studied by Svegar had clinical features of liver disease (Aagenaes, Fagerhol, Elgjo, Munthe, and Hovig, 1974).

Distinctive sharply defined, diastase-resistant periodic acid Schiff (PAS) positive globules were described by Sharp in 1971 in the cytoplasm of periportal hepatocytes of patients with $\alpha_{1}$-ATD. Similar globules, when examined by immunofluorescence after staining with a fluoresceinconjugated specific antiserum to $\alpha_{1}$-antitrypsin, react as if they were accumulations of the antiprotease (De Lellis, Balogh, Merk, and Chirife, 1972). Electron microscopy by the latter authors and immuno-peroxidase electron microscopy by Aagenaes et al (1974) show that the material accumulates in cisternae of the rough endoplasmic reticulum of hepatocytes. Another feature stated by Aagenaes, Matlary, Elgjo, Munthe, and Fagerhol (1972) to be characteristic of neonatal cholestatis in the PiZZ state was the presence of finely granular greyishbrown pigment in the periportal hepatocytes. 
In the course of histological examination of liver biopsy specimens from infants with neonatal hepatitis syndrome PAS-positive material had been noticed only sporadically in PiZZ individuals, throwing doubt on its value in the diagnosis of the $\alpha_{1}$-ATD state. We have therefore investigated the specificity and frequency of this histological finding. We have also looked for the granular pigment noted by Aagenaes et al (1972) and any other features which might be specific to hepatitis occurring in infants with $\alpha_{1}$-ATD.

\section{Methods}

Sixty-six liver biopsies and three postmortem liver specimens from 53 children were examined histologically without knowledge of the PiZZ state. The infants formed part of a well documented on-going study into aetiological and prognostic factors in neonatal hepatitis syndrome in south-east England (Porter et al, 1972; Cottrall et al, 1974). The age at which biopsies were performed is shown (fig 1). After fixation in $10 \%$ formol saline and preparation of paraffin sections of liver tissue the histological sections were examined stained with Ehrlich's haematoxylin and eosin (HE), Gordon and Sweet's silver impregnation of reticulin, PAS, and Perls's technique. Other stains used were the Ziehl-Neelsen method modified for ceroid (ZN), Schmorl's method for reducing substances including lipofuscin, and Fouchet's method for bilirubin. $\alpha_{1}$-Antitrypsin was estimated by a modified acid starch gel method (Porter et al, 1972) and found to be deficient, with PiZZ characteristics, in sera from 14 of the 53 children.

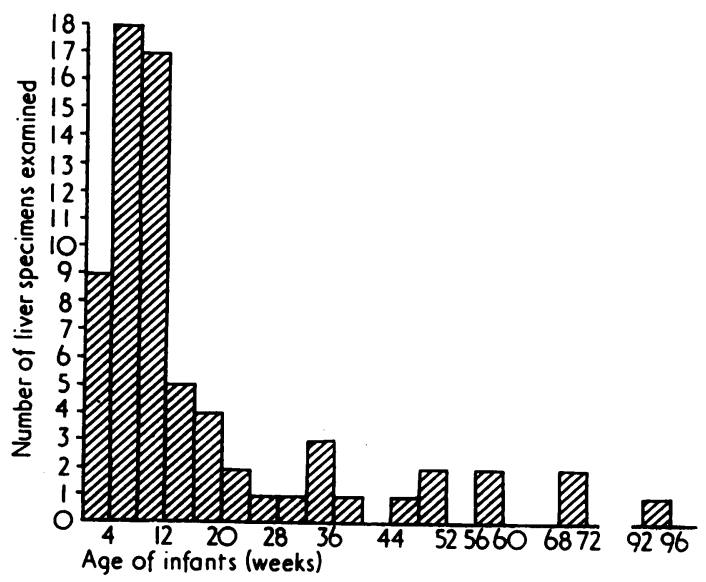

Fig 1 Number of liver biopsies examined and age at time of biopsy.

\section{Results}

Twelve children (17 biopsies) were eventually found $\stackrel{\vec{F}}{\stackrel{7}{7}}$ at exploratory laparotomy to have biliary atresia, $\frac{}{0}$ although this was not always apparent from their initial liver biopsies which were taken at an average age of 13 weeks (range $2 \frac{1}{2}$ to 32 weeks). None of $\frac{\mathbb{Q}}{\alpha}$ these 12 was of PiZZ phenotype. There was considerable variation in the histological features seen in the liver of the remaining 41 children. In view of the lack of a standard terminology to describe biopsy findings in infantile liver disease, terms employed primarily to changes seen in liver disease in the adult (Scheuer, 1973) have been used in some instances. It was decided to assign each biopsy to one of nine categories according to the predominant histological feature present, as shown in the table. In no case was liver histology normal at the initial biopsy, although there was a return to normality on the second biopsy in one child without $\alpha_{1}$-ATD. The picture of classical neonatal hepatitis with marked giant-cell transformation of hepatocytes was seen in 17 PiMM infants but in only two of 15 samples of liver tissue of PiZZ infants. Six biopsies of the PiMM infants and one of a PiZZ infant showed histological features similar to those of acute cholestatic hepatitis in the adult, with bile retention in hepatocytes and bile canaliculi, many single eosinophilic necrotic hepatocytes ('acidophil bodies') and an infiltrate of lymphocytes in portal tracts, but with virtually no giant-cell transformation. An acute hepatitis, with similar hepatocellular and portal tract changes but without prominent cholestasis, was seen in three biopsies of PiZZ infants, a picture not found in any PiMM infants. In three cases, all $\alpha_{1}$-antitrypsin deficient, cholestatic hepatitis was accompanied by glandular transformation of periportal hepatocytes and prominent dilated periportal ductular structures in the location of canals of Hering. An appearance resembling chronic persistent hepatitis in the adult, with only a rather scanty lymphocytic infiltration of portal tracts, was seen in five PiZZ children and in just one PiMM phenotype. Evidence of more active inflammation, with a denser infiltrate of lymphocytes and conspicuous fibrosis with expansion of portal tracts was seen in the initial liver biopsies of two children with PiMM phenotype and in follow-up biopsies of two PiZZ individuals. Three of the 14 children of PiZZ phenotype have developed cirrhosis compared with four of the 27 children of normal phenotype. The cirrhosis in most cases was of micronodular type but in two of the children with $\alpha_{1}$-ATD a secondary biliary type cirrhosis was present. (One child with secondary biliary cirrhosis (case 1 , fig 4) and the PiZZ infant with micronodular cirrhosis (case 4) 


\section{HISTOLOGICAL FEATURES}

Normal

Neonatal hepatitis with giant cell transformation

Neonatal hepatitis without giant cell transformation (similar to acute viral hepatitis in adult)

Picture similar to cholestatic hepatitis in adult

Cholestatic hepatitis with periportal glandular and ductular structures

Picture similar to chronic persistent hepatitis in adult

Chronic inflammation and conspicuous fibrous expansion of portal tracts

Micronodular cirrhosis

Secondary biliary cirrhosis
BIOPSIES OF PEZ INFANTS BIOPSIES OF PIMM INFANTS

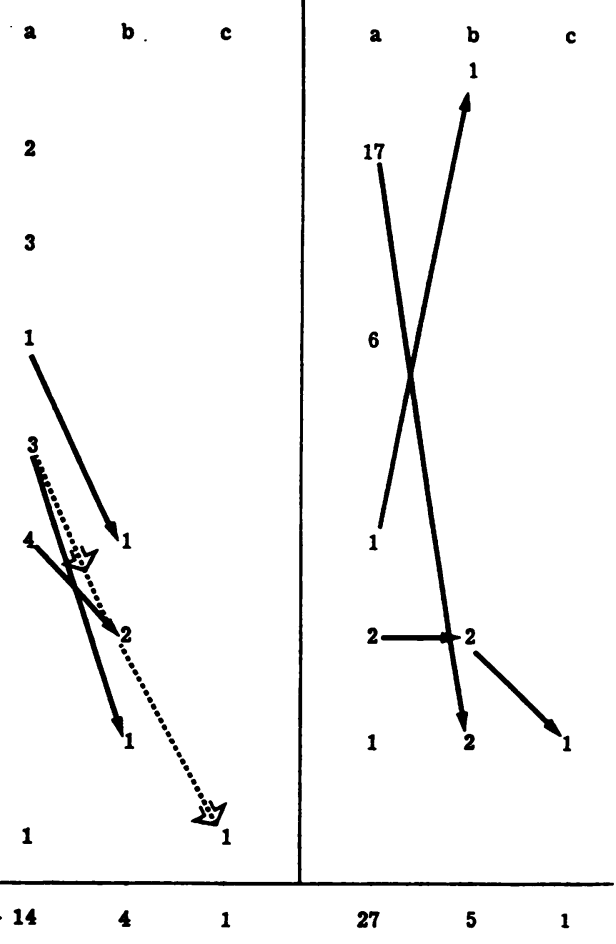

Table Principal histological features in liver biopsies of PiZZ and PiMM individuals without biliary atresia. Column a-first biopsy; column b-second biopsy; column c-third biopsy. Arrows link repeat biopsies, indicating change in pathological categories in individual cases.

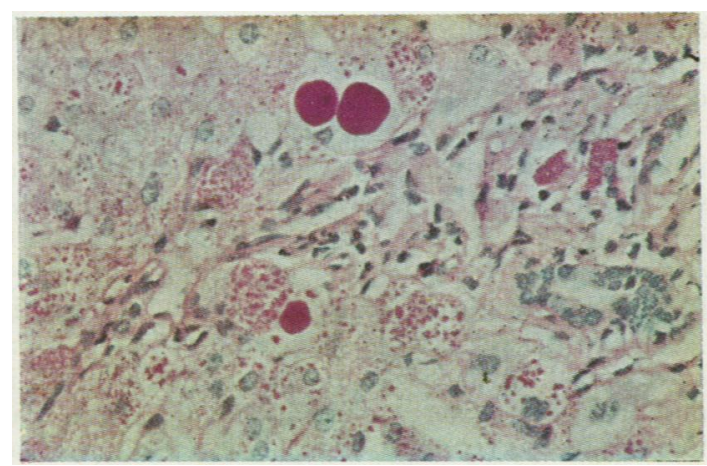

Fig 2

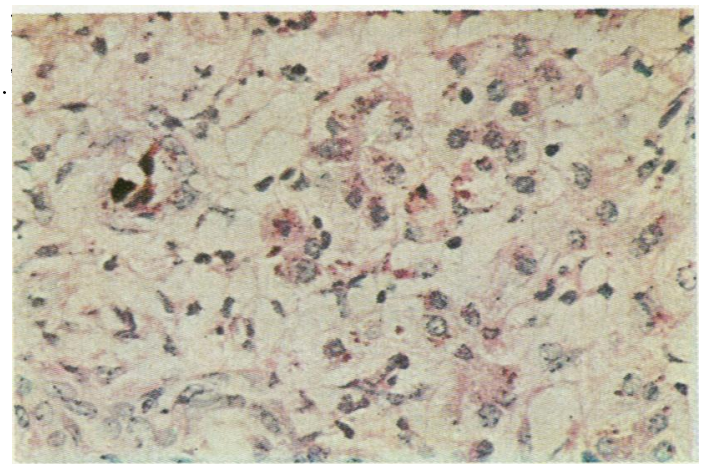

Fig 3

Fig 2 Section of liver biopsy of infant with $a_{1}-A T D$ aged 18 weeks (case 13) showing diastase-resistant, PAS-positive, pure magenta-coloured, sharply defined globules of varying size in the cytoplasm of periportal hepatocytes. PAS stain, after diastase $\times 800$.

Fig 3 The edge of a portal tract from an infant aged 4 months with biliary atresia, PiMM phenotype. Periportal hepatocytes contain diastase resistant, PAS-positive material, which is poorly demarcated and brownish-purple in colour. A ductule at the edge of the portal tract is distended by bile and there is considerable feathery degeneration of hepatocytes. Surgical liver biopsy. PAS after diastase $\times 800$. 
have previously been reported by Porter et al (1972)).

Diastase-resistant, PAS-positive, pure magentacoloured, sharply defined globules between 2 and 20 microns in diameter, similar to those described by Sharp (1971) and by De Lellis et al (1972), were found in periportal and paraseptal hepatocytes in a total of 10 of the liver biopsies from eight of the infants (fig 2). Sera from all eight of these infants were $\alpha_{1}$-antitrypsin deficient. In one further child, not deficient in serum $\alpha_{1}$-antitrypsin, but with biliary atresia, sparse, less well defined PAS-positive globules were found in some periportal hepatocytes showing feathery degeneration associated with marked cholestasis (fig 3).

It should be stressed that the sharply defined globules described above are distinct from the irregular fragments of diastase-resistant, PASpositive, brownish-purple material in periportal Kupffer cells and in portal tract macrophages found in many of the liver biopsies irrespective of the Pi state of the infant. The latter material stained positively with Schmorl's and ZN stains, suggesting that it was a mixture of lipofuscins. Figure 4 lists the age of the children at biopsy who were found to be $\alpha_{1}$-antitrypsin deficient and indicates whether or not PAS-positive globules were found histologically in their livers. It will be noted that in 10 biopsies, all from infants 13 weeks old or less, no PAS-positive globules were found and that globules were seen only in infants aged 12 weeks or more.

We found finely granular, greyish-brown pigment in HE stained sections, as described by Aagenaes et al (1972), in all but three of the liver biopsies of $\alpha_{1}$-antitrypsin deficient children. This pigment did not appear to be specific, however, as it stained strongly by Perls' method, and rather more variably

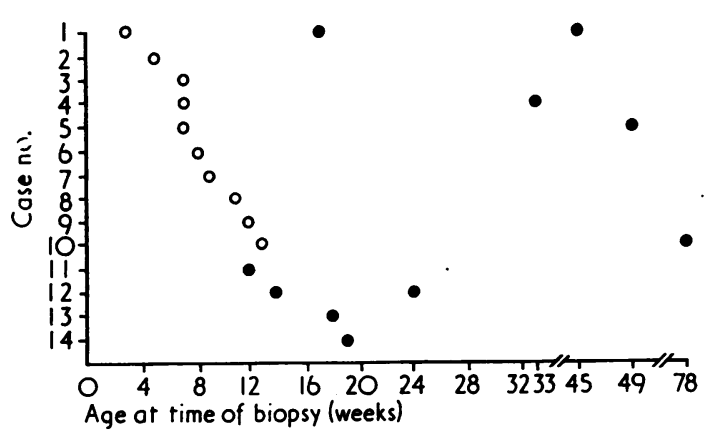

Fig 4 Diagram showing the age of the 14 infants of PiZZ phenotype at the time of liver biopsy, indicating those biopsies in which PAS-positive globules similar to those shown in fig 3 were seen: open circles, no PAS-positive globules found; closed circles, PAS-positive globules present. and weakly by Schmorl's, ZN, and modified Fouchet's techniques, indicating that these granules are predominantly haemosiderin with some admixture of lipofuscins and bile. Similar pigment granules were also seen in 24 of 27 initial biopsies of the cases who were not $\alpha_{1}$-antitrypsin deficient.

There was no significant age difference between the PiZZ and PiMM children at initial liver biopsy, the average age in both groups being $10 \frac{1}{2}$ weeks.

\section{Discussion}

Our studies show that discrete, sharply defined PAS-positive globules in liver parenchymal cells are a useful diagnostic feature of $\alpha_{1}$-antitrypsin deficiency in the infant of more than 12 weeks of age. The hepatocytes which contain these globules appear otherwise normal. Such globules seem to be specific for the deficiency state in the histological material examined. It must be emphasized, however, that other PAS-positive material is not uncommonly found in liver tissue of infants with hepatitis. This material is to be found in Kupffer cells and in macrophages within the portal tracts and bears no relationship to the $\mathrm{Pi}$ phenotype of the infant. Further, PAS-positive material was found within hepatocytes showing feathery degeneration in association with periportal cholestasis in two biopsies from one of 12 patients with biliary atresia. Below the age of 12 weeks the diagnosis of $\alpha_{1}$-ATD cannot be made on histological grounds alone; we have noted the fine greyish-brown granules in hepatocytes, as described by Aagenaes et al, but find that they are not specific as they consist of haemosiderin with some lipofuscins and bilirubin and are commonly seen in neonatal hepatitis, whether or not the children are $\alpha_{1}$-antitrypsin deficient.

No qualitatively unique histological picture was found in PiZZ individuals. Giant cell transformation was less frequent and less marked than in the PiMM individuals. In contrast, other histological evidence of more severe damage was seen more commonly in the PiZZ group; features of acute hepatitis similar to those in adults, glandular transformation of periportal hepatocytes or chronic inflammation of portal tracts were recorded in $70 \%$ of initial biopsies in this group as opposed to only $4 \%$ of the PiMM patients. We cannot comment on the final frequency of cirrhosis in the two groups of patients, but the finding of cirrhosis in three of $14 \alpha_{1}$-ATD children, as opposed to four of 27 subjects with PiMM phenotype suggests that the prognosis of neonatal hepatitis syndrome may be worse in patients with $\alpha_{1}$-ATD. Clearly, further follow-up studies will be necessary to verify this. 
The failure to find PAS-positive globules under the age of 12 weeks suggests that until this age insufficient glycoprotein has accumulated in the hepatocytes to be histologically detectable; this may support the conclusion of De Lellis et al (1972), based on immunofluorescent studies confirmed by Aagenaes et al (1972) and ourselves, that the globules represent retained, accumulated anti-protease within the liver cells where it is synthesized but for some reason cannot be released. Why these aggregates should become evident at 3 months of age is not clear. Serum $\alpha_{1}$-antitrypsin levels in newborn infants are normal, even in those born pre-term (El-Bardeesy, Johnson, and Bissett, 1972). It has been postulated that these accumulations result from the synthesis of a structurally different protein (Aagenaes et al, 1974). The finding that $\alpha_{1}$-antitrypsin in sera of PiZZ individuals lacks two of the six sialic acid residues found in $\alpha_{1}$-antitrypsin of PiMM individuals (Cox, 1973) suggests that a more minor modification of the carbohydrate portion of the glycoprotein, affecting the ability of the hepatocyte to secrete $\alpha_{1}$-antitrypsin, could be responsible.

The pathogenesis of liver disease in the condition is still obscure. Current evidence suggests that the local accumulation of the glycoprotein protease inhibitor is not the cause of liver damage since similar globules have been found in both PiZZ and PiSS individuals without liver disease (Gordon, Dixon, Rogers, Mittman, and Lieberman, 1972) and are absent before 12 weeks of age in our series. It has been suggested that $30 \%$ of PiZZ individuals are susceptible to liver disease (Aagenaes et al, 1972), and although Svegar's study (Aagenaes et al, 1974) indicates that this may be an underestimate, it would appear that an additional, possibly exogenous, factor is necessary to produce liver disease. Hepatitis B antigen was considered to be such a factor in some cases in this present series. A factor acting in utero, possibly genetic or environmental, is suggested by the low birth weights of earlier reported cases. How such an agent may cause ongoing liver damage is not known. The most favoured hypothesis relates the liver (and lung) disease to a limitation of inhibition of proteases in the tissues concerned. Careful prospective clinicopathological studies may help to clarify the picture. Further biochemical studies of the synthesis, hepatic secretion, and physiological role of $\alpha_{1}$-antitrypsin will probably be necessary to explain our observations and to enable one to understand the mechanism of liver disease in individuals with serum deficiency of this anti-protease.

Pi phenotyping was carried out by Dr P. J. L. Cook, MRC Human Biochemical Genetics Unit, University College Hospital, London WC1. Technological assistance was provided by Mr George Harwood, Department of Morbid Anatomy, King's College Hospital.

We are grateful for the support and encouragement of Professor E. A. Wright and Professor C. E. Stroud.

Financial assistance was provided by the Wellcome Foundation.

\section{References}

Aagenaes, Ø., Fagerhol, M., Elgjo, K., Munthe, E., and Hovig, T. (1974). Pathology and pathogenesis of liver disease in $\alpha_{1}$-antitrypsin deficient individuals. Postgrad. med. J., 50, 365-375.

Aagenaes, Ø., Matlary, A., Elgjo, K., Munthe, E., and Fagerhol, M. (1972). Neonatal cholestasis in $\alpha_{1}$-antitrypsin deficient children. Acta paediat. scand., 61, 632-642.

Brunt, P. W. (1974). Progress report. Antitrypsin and the liver. Gut, 15, 573-580.

Cottrall, K., Cook, P. J. L., and Mowat, A. P. (1974). Neonatal hepatitis syndrome and $\alpha_{1}$-antitrypsin deficiency: an epidemiological study in south-east England. Postgrad. med. J., 50, 376-380.

Cox, D. W. (1973). Defect in $\alpha_{1}$-antitrypsin deficiency. (Letter.) Lancet, 2, 844-845.

DeLellis, R. A., Balogh, K., Merk, F. B., and Chirife, A. M. (1972). Distinctive hepatic cell globules in adult $\alpha_{1}$-antitrypsin deficiency. Arch. Path., 94, 308-316.

El-Bardeesy, M. W., Johnson, A. M., and Bissett, L. S. (1972). Serum proteinase inhibitors in infants with hyaline membrane disease. J. Pediat., 81, 579-587.

Gordon, H. W., Dixon, J., Rogers, J. C., Mittman, C., and Lieberman, J. (1972). $\alpha_{1}$-Antitrypsin accumulation in livers of emphysematous patients with $\alpha_{1}$-antitrypsin deficiency. Hum. Path., 3, 361-370.

Hutchison, D. C. S., Cook, P. J. L., Barter, C. E., Harris, H., and Hugh-Jones, P. (1971). Pulmonary emphysema and $\alpha_{1}$-antitrypsin deficiency. Brit. med. J., 1, 689-694.

Porter, C. A., Mowat, A. P., Cook, P. J. L., Haynes, D. W. G. Shilkin, K. B., and Williams, R. (1972). $\alpha_{1}$-Antitrypsin deficiency and neonatal hepatitis. Brit. med. J., 3, 435-439.

Scheuer, P. J. (1973). Liver Biopsy Interpretation, 2nd edition. Bailliere, Tindall, London.

Sharp, H. L. (1971). $\alpha_{1}$-Antitrypsin deficiency. Hosp. Pract., $5,83$.

Sharp, H. L., Bridges, R. A., Krivit, W., and Freier, E. F. (1969). Cirrhosis associated with $\alpha_{1}$-antitrypsin deficiency: a previously unrecognised inherited disorder. J. Lab. clin. Med., 73, 934-939. 
and 4 are two very extensive tables occupying between them almost half of the monograph. One is a list of species held at the NCTC with details of steps in their freeze-drying, and the other gives the viability of some freeze-dried bacteria. There is a section on the formulae of media used and a very extensive and useful bibliography.

W. J. RYAN

Practical Haematology, 5th edition. By J. V. Dacie and S. M. Lewis. (Pp. vii + 629 ; illustrated; $£ 6 \cdot 00$.) London:Churchill Livingstone. 1975.

Everyone will welcome the new edition of this manual which is now the accepted standard text for haematology laboratories at home as well as overseas. As in previous editions the techniques and schemes of investigation described are those practised at the Hammersmith Hospital and the Royal Postgraduate Medical School. Although the general scope and organization of the book remains much as it was in previous editions, there is much valuable new material. The use of SI units throughout the book will undoubtedly help the growing pains which some hospitals are experiencing in changing over to the International System of Units. Various specialists, most of whom work or have worked at the Royal Postgraduate Medical School, have collaborated in the different sections, notably the chapters dealing with blood groups, haemostatic disorders, hereditary haemolytic anaemias, megaloblastic and iron-deficiency aenaemias, auto-immune haemolytic anaemias, and haemoglobinopathies respectively. One entirely new chapter entitled Leucocyte and Platelet Antigens and Antibodies by Dr. Sylvia D. Lawler has been included which is particularly relevant to tissue transplantation. Although the existing book has been thoroughly revised and brought up to date, the authors must be congratulated on keeping it to a manageable size. Everyone who works in a haematology laboratory should have his own copy. Let us hope that the price of $£ 6.00$ is not too much of a deterrent.

KATHERINE M. DORMANDY

Salivary Glands in Health and Disease. By D. K. Mason and D. M. Chisholm. (Pp. vii +320 ; illustrated; $£ 8.50$.) London: W. B. Saunders. 1975.

This book is presented as a review of salivary glands and the methods used in their study in health and disease. Early chapters on basic aspects of the subject are useful, and the histopathologist will find, in particular, the section on histology and histochemistry detailed and up to date. A few pages follow on history and clinical examination, probably inserted for completeness; some of the 10 clinical photographs seem superfluous, such as the one depicted as 'bimanual palpation' of the parotid showing the hands placed externally on both sides of the face. In the interesting chapter on changes in the chemical composition of saliva, the alterations in fibrocystic disease of the pancreas and in thyroid disease will intrigue many pathologists. The histopathologist will not get much help from this book when he has problem sections of salivary neoplasms, but the account of Sjögren's syndrome is detailed and authoritative, and I found the counterposing chapter on the lympho-epithelial lesion clear and well reasoned.

This is a good review in the multidisciplinary mode, a life-style which pathologists must adopt in order to survive in modern medicine. Chemical pathologists and histopathologists will find much of practical value in it.

\section{MICHAELS}

Biology of Cancer, 2nd revised edition. Edited by E. J. Ambrose and F. J. C. Roe. (Pp. 315; illustrated; £15.00.) Chichester: John Wiley. 1975.

The current modern ideas in cancerviral aetiology, immunotherapy, genetic change, environmental carcinogenesiswere all topical in 1909 when Paul Ehrlich (quoted here by Peter Alexander) outlined the concept of immunosurveillance. We have since documented more precisely the properties of the cancer cell and investigated in detail carcinogenic agencies but we still fall short of a real understanding of the neoplastic process. This book summarizes present knowledge and does it well though there are two omissions. There is no consideration of hormones and radiation is scarcely mentioned. All the chapters are by experts but the approach varies. Ambrose's account of the cell surface is highly individual while Carter's chapter on metastasis is an excellent brief introduction. Systemic factors produced by human tumours are neatly dealt with by Neville and Symington, and it is good to see ectopic hormone production and oncofetal antigens dealt with as expressions of the same phenomenon. Chapters by Roberts on nucleic acids and by Rowson on viruses are good accounts and give prodigeous reference lists. Alexander's chapter on immunity concentrates on escape from host control and is well done but a broader approach might have been better. Not a book for the specialist but a basic text where anyone interested in cancer can find background and references on the biology of cancer.

D. G. HARNDEN

\section{Correction}

Liver disease in infancy: histological features and relationship to $a_{1}$-antitryspin phenotype. J. clin. Path., 1975, 28, 559.

I much regret that in the above paper we erroneously stated the incidence of liver disease in the $40 \mathrm{Pi}$ type $\mathrm{ZZ}$ infants studied by Svegor to be 34; in fact the correct figure is $3-4(10 \%)$ (Aagenaes, Fagerhol, Elgjio, Munther, and Hovig, Postgrad. med. J., 1974, 50, 365-375).

I. C. TALBOT

Department of Pathology University of Leicester 